TRANSACTIONS OF THE

AMERICAN MATHEMATICAL SOCIETY

Volume 361, Number 6, June 2009, Pages 2913-2927

S 0002-9947(09)04929-0

Article electronically published on January 22, 2009

\title{
COMPUTABILITY, NONCOMPUTABILITY AND UNDECIDABILITY OF MAXIMAL INTERVALS OF IVPS
}

\author{
D.S. GRAÇA, N. ZHONG, AND J. BUESCU
}

\begin{abstract}
Let $(\alpha, \beta) \subseteq \mathbb{R}$ denote the maximal interval of existence of solutions for the initial-value problem

$$
\left\{\begin{array}{l}
\frac{d x}{d t}=f(t, x) \\
x\left(t_{0}\right)=x_{0}
\end{array}\right.
$$

where $E$ is an open subset of $\mathbb{R}^{m+1}, f$ is continuous in $E$ and $\left(t_{0}, x_{0}\right) \in E$. We show that, under the natural definition of computability from the point of view of applications, there exist initial-value problems with computable $f$ and $\left(t_{0}, x_{0}\right)$ whose maximal interval of existence $(\alpha, \beta)$ is noncomputable. The fact that $f$ may be taken to be analytic shows that this is not a lack of the regularity phenomenon. Moreover, we get upper bounds for the "degree of noncomputability" by showing that $(\alpha, \beta)$ is r.e. (recursively enumerable) open under very mild hypotheses. We also show that the problem of determining whether the maximal interval is bounded or unbounded is in general undecidable.
\end{abstract}

\section{INTRODUCTION}

In the last decades, digital computers have evolved at a breathtaking speed. Nowadays, with the availability of fast, cheap, and reliable computers, their widespread use has become transversal to all fields of science. In particular, areas of mathematics such as numerical analysis have greatly benefited from their systematic use. Surprisingly, relatively little theoretical work exists relating these areas to computability theory. This theory emerged in the 1930s from the groundwork of logicians such as Gödel, Turing, Church, Kleene, and Post, among others. In this formal setting, computations are performed by Turing machines, first introduced by Turing in 23 and accepted by the scientific community as the standard model of computation. In practice, the Turing machine (TM for short) is capable of solving

Received by the editors July 6, 2006.

2000 Mathematics Subject Classification. Primary 65L05, 68Q17; Secondary 03D35.

The first author thanks M. Campagnolo, O. Bournez, and E. Hainry for helpful discussions. The first author was partially supported by Fundação para a Ciência e a Tecnologia and EU FEDER POCTI/POCI via CLC, grant SFRH/BD/17436/2004, and the project ConTComp POCTI/MAT/45978/2002. Additional support was also provided by the Fundação Calouste Gulbenkian through the Programa Gulbenkian de Estímulo à Investigação.

The second author was partially supported by University of Cincinnati's Taft Summer Research Fellowship.

The third author was partially supported by Fundação para a Ciência e a Tecnologia and EU FEDER POCTI/POCI via CAMGSD. 
exactly the same problems as an ordinary computer (as long as enough memory is provided).

One of the great achievements of computability theory is to show that there are noncomputable (or undecidable) problems, i.e. problems that cannot be solved with the use of a Turing machine. The best-known examples of noncomputable problems are the Halting Problem and Hilbert's 10th Problem [22].

However, the Turing machine cannot be applied directly to deal with real quantities because it can only have as input and output a "finite number of bits". To circumvent this problem, several extensions of the Turing machine model have been proposed. One such extension is the BSS model [5], 4, which instead of using a "finite number of bits" allows the use of a finite number of reals. Computations over the reals are then performed using infinite-precision arithmetic. While this model is elegant, especially from the algebraic point of view, its relevance to "practical computations" may be limited since, in general, bounded precision arithmetic is used. In this context, we prefer to use the computable analysis theory, whose foundations go back to Banach, Mazur [15, Grzegorczyk [10, and Lacombe [13, where an approximation of the output with arbitrary precision is computed from a suitable approximation of the input. For more recent developments in computable analysis, the reader is referred to [19, [12, 24, 6], [7.

In this paper, we study computability and noncomputability in initial-value problems (henceforth abbreviated to IVPs) of ordinary differential equations based on the model of computable analysis. It is well known that ordinary differential equations are fundamental in modeling physical processes. The most general ODE is written as a system $\dot{x}=f(t, x)$, where $E$ is an open subset of $\mathbb{R}^{m+1}, f: E \rightarrow \mathbb{R}^{m}$, $x=x(t) \in \mathbb{R}^{m}$ is a function of $t$ and $\dot{x}$ denotes the derivative of $x$ with respect to $t$. The well-posedness of such systems follows from the basic existence-uniqueness theory [8], [14]; in particular, if $f$ is continuous on $E$ and locally Lipschitz in the second argument, the initial-value problem (IVP)

$$
\left\{\begin{array}{l}
\dot{x}=f(t, x) \\
x\left(t_{0}\right)=x_{0}
\end{array}\right.
$$

has a unique solution $x(t)$ defined on a maximal interval of existence $I=(\alpha, \beta) \subset \mathbb{R}$ that is continuously differentiable on $I$. In general, however, it is not possible to solve the above nonlinear system analytically with an explicit solution formula. Many of those nonlinear systems can only be solved numerically on computers. Numerical methods are usually tailor-made for individual problems and often depend on certain assumptions, for example, the existence of some time interval where the solution is defined. This requirement is crucial but in general hard to verify.

The satisfactory solution is to have some "automated method" that determines the maximal interval $(\alpha, \beta)$ and computes the solution on $(\alpha, \beta)$ from the data defining the IVP (1.1). Thus, it becomes useful to know whether it is possible to derive such an "automated method". In this paper, we present a negative answer to the question. We show that the maximal interval where the solution of the IVP (1.1) is defined may not be computable, even when the function $f$ is analytic as well as computable and the initial condition is computable. In such circumstances, there is no algorithm to decide, given an arbitrary time $t$ as input, whether or not $t \in(\alpha, \beta)$. The undecidability indicates that the limit behavior of the IVP (1.1) may not be determined by "general numerical recipes". In other words, such undecidability suggests limitations concerning numerical methods for solving ODEs. 
There are other noncomputability results related to the initial-value problems of differential equations. For example, Pour-El and Richards [17] showed that the IVP (1.1) defined with computable data may have noncomputable solutions. In [18, 20] it is shown that there is a three-dimensional wave equation, defined with computable data, such that the unique solution is nowhere computable. However, in these examples, noncomputability is not "genuine" in the sense that the problems under study are ill-posed: either the solution is not unique or it is unstable 25. In other words, ill-posedness was at the origin of noncomputability in those examples. In contrast, all IVPs studied in this paper are classically well-posed and, consequently, the noncomputability results associated to these well-posed problems reflect the true computational deficiency inherited by the problems. For reference we also mention the existence of other results about computability of ODEs that can be found in [1], 2], 3], 11, [9, 12], 21].

The paper is organized as follows. Section 2 introduces necessary concepts and results from computability theory, computable analysis and the theory of ODEs. Section 3 presents a theorem stating that the maximal interval $(\alpha, \beta) \subseteq \mathbb{R}$ of the IVP (1.1) where the solution is defined is recursively enumerable and the solution is computable on $(\alpha, \beta)$, provided the data defining the initial-value problem is computable. Section 4 contains two counterexamples showing that the maximal interval $(\alpha, \beta)$ is r.e. but not necessarily computable. The first example treats the case where the ODE is defined by a continuous and computable function, while the second deals with the case where the ODE is defined by an analytic as well as computable function. Finally, Section 5 shows that even the problem of deciding whether the maximal interval is bounded or not is undecidable.

\section{Preliminaries}

This section introduces necessary concepts and results from computability theory, computable analysis and from the theory of ODEs. For more details the reader is referred to [22 for computability theory, [19], [12, 24] for computable analysis, and [8], 14] for ODEs.

We assume that the reader is familiar with the notion of TMs (cf. [22]). Roughly speaking, a TM can be treated as a computer program written in one's favorite programming language. We turn now to the basic computability definitions. Denote by $\mathbb{N}, \mathbb{Q}$ and $\mathbb{R}^{n}$ respectively the set of natural numbers containing 0 , the set of rational numbers, and $n$-dimensional Euclidean space.

Definition 2.1. (1) A function $f: \mathbb{N}^{n} \rightarrow \mathbb{N}$ is computable (or recursive) if there is a TM, which on input $\left(a_{1}, \ldots, a_{n}\right) \in \mathbb{N}^{n}$ outputs $f\left(a_{1}, \ldots, a_{n}\right)$.

(2) The set $L \subseteq \mathbb{N}^{n}$ is computable or decidable if the characteristic function $\chi_{L}: \mathbb{N}^{n} \rightarrow\{0,1\}$ defined by $\chi_{L}(x)=1$ if $x \in L, \chi_{L}(x)=0$ otherwise, is computable.

(3) The set $L \subseteq \mathbb{N}$ is recursively enumerable (r.e. for short) if there is a computable function $f: \mathbb{N} \rightarrow \mathbb{N}$ such that $L=\{f(i): i \in \mathbb{N}\}$.

It is well known that there are r.e. sets which are not recursive. Indeed, it can be shown that a set $A$ is recursive iff both $A$ and its complement $A^{c}$ are r.e. [16]. The above Turing machine model can be extended to deal with computations that operate on real-valued data. Among different extensions, we use the computable analysis approach. In this approach, informally, a function $f: \mathbb{R} \rightarrow \mathbb{R}$ is computable 
if there is a computer program that does the following. Let $x \in \mathbb{R}$ be an arbitrary element in the domain of $f$. Given an output precision $2^{-n}$, the program has to compute a rational approximation of $f(x)$ with precision $2^{-n}$.

To formalize this notion, we need oracle TMs. We say that M is an oracle TM if, at any step of the computation of M using the oracle $\phi: \mathbb{N} \rightarrow \mathbb{N}^{k}$, M is allowed to query the value $\phi(n)$ for any $n$.

Definition 2.2. (1) A sequence $\left\{r_{n}\right\}$ of rational numbers is called a $\rho$-name of a real number $x$ if there are three functions $a, b$ and $c$ from $\mathbb{N}$ to $\mathbb{N}$ such that for all $n \in \mathbb{N}, r_{n}=(-1)^{a(n)} \frac{b(n)}{c(n)+1}$ and

$$
\left|r_{n}-x\right| \leq \frac{1}{2^{n}} .
$$

(2) A real number $x$ is called computable if it has a computable $\rho$-name, i.e., if $a, b$ and $c$ in (2.1) are computable (recursive) functions.

(3) A sequence $\left\{x_{k}\right\}_{k \in \mathbb{N}}$ of real numbers is computable if there are three computable functions $a, b, c$ from $\mathbb{N}^{2}$ to $\mathbb{N}$ such that, for all $k, n \in \mathbb{N}$,

$$
\left|(-1)^{a(k, n)} \frac{b(k, n)}{c(k, n)+1}-x_{k}\right| \leq \frac{1}{2^{n}} .
$$

The notion of the $\rho$-name can be extended to points in $\mathbb{R}^{l}$ as follows: a sequence $\left\{\left(r_{1 n}, r_{2 n}, \ldots, r_{l n}\right)\right\}_{n \in \mathbb{N}}$ of rational vectors is called a $\rho$-name of $x=\left(x_{1}, x_{2}, \ldots, x_{l}\right)$ $\in \mathbb{R}^{l}$ if $\left\{r_{j n}\right\}_{n \in \mathbb{N}}$ is a $\rho$-name of $x_{j}, 1 \leq j \leq l$. Similarly, one can define computable points and sequences over $\mathbb{R}^{l}, l>1$, by assuming that each component is computable. Next we present a notion of computability for open and closed subsets of $\mathbb{R}^{l}$ (cf. 24], Definition 5.1.15).

Definition 2.3. (1) An open set $E \subseteq \mathbb{R}^{l}$ is called recursively enumerable (r.e. for short) open if there are computable sequences $\left\{a_{n}\right\}$ and $\left\{r_{n}\right\}, a_{n} \in E$ and $r_{n} \in \mathbb{Q}$ such that

$$
E=\bigcup_{n=0}^{\infty} B\left(a_{n}, r_{n}\right)
$$

Without loss of generality one can also assume that for any $n \in \mathbb{N}$, the closure of $B\left(a_{n}, r_{n}\right)$, denoted as $\overline{B\left(a_{n}, r_{n}\right)}$, is contained in $E$, where $B\left(a_{n}, r_{n}\right)$ $=\left\{x \in \mathbb{R}^{l}:\left|x-a_{n}\right|<r_{n}\right\}$.

(2) A closed subset $K \subseteq \mathbb{R}^{l}$ is called r.e. closed if there exist computable sequences $\left\{b_{n}\right\}$ and $\left\{s_{n}\right\}, b_{n} \in \mathbb{Q}^{l}$ and $s_{n} \in \mathbb{Q}$, such that $\left\{B\left(b_{n}, s_{n}\right)\right\}_{n \in \mathbb{N}}$ lists all rational open balls intersecting $K$.

(3) An open set $E \subseteq \mathbb{R}^{l}$ is called computable (or recursive) if $E$ is r.e. open and its complement $E^{c}$ is r.e. closed. Similarly, a closed set $K \subseteq \mathbb{R}^{l}$ is called computable (or recursive) if $K$ is r.e. closed and its complement $K^{c}$ is r.e. open.

It is well known that an open interval $(\alpha, \beta) \subset \mathbb{R}$ or a closed interval $[\alpha, \beta]$ is computable if and only if $\alpha$ and $\beta$ are computable real numbers. Having defined the notion of recursive and r.e. sets, we are now ready to introduce the notion of computable functions defined on those sets [19, 12, 24]. 
Definition 2.4. Let $A \subseteq \mathbb{R}^{l}$ be either an r.e. open set or an r.e. closed set.

(1) A function $f: A \rightarrow \mathbb{R}^{m}$ is computable if there is an oracle Turing machine such that for any input $n \in \mathbb{N}$ (accuracy) and any $\rho$-name of $x \in A$ given as an oracle, the machine will output a rational vector $r$ satisfying $|r-f(x)| \leq$ $2^{-n}$

(2) A sequence $\left\{f_{i}\right\}_{i \in \mathbb{N}}$ of functions, where $f_{i}: A \subseteq \mathbb{R}^{l} \rightarrow \mathbb{R}^{m}$, is computable if there is an oracle Turing machine such that for any input $n \in \mathbb{N}$ (accuracy), any $i \in \mathbb{N}$, and any $\rho$-name of $x \in A$ given as an oracle, the machine will output a rational vector $r$ satisfying $\left|r-f_{i}(x)\right| \leq 2^{-n}$.

Recall that a function $f: E \rightarrow \mathbb{R}^{m}, E \subseteq \mathbb{R}^{l}$, is said to be locally Lipschitz on $E$ if it satisfies a Lipschitz condition on every compact set $V \subset E$. The following definition gives a computable analysis analog of this condition.

Definition 2.5. Let $E=\bigcup_{n=0}^{\infty} B\left(a_{n}, r_{n}\right) \subseteq \mathbb{R}^{l}$, where $\overline{B\left(a_{n}, r_{n}\right)} \subseteq E$, be an r.e. open set. A function $f: E \rightarrow \mathbb{R}^{m}$ is called effectively locally Lipschitz on $E$ if there exists a computable sequence $\left\{K_{n}\right\}$ of positive integers such that

$$
|f(x)-f(y)| \leq K_{n}|x-y| \text { whenever } x, y \in \overline{B\left(a_{n}, r_{n}\right)} .
$$

Strictly speaking, it would only be necessary to require in the above definition that the sequence $\left\{K_{n}\right\}$ be computable and formed by positive reals. However, given a (computable) local Lipschitz constant $L_{n}$ for a compact set, any integer $K_{n} \geq L_{n}$ is also a local Lipschitz constant for that set, and therefore there is no loss of generality in assuming that the sequence $\left\{K_{n}\right\}$ in Definition 2.5 consists only of integers.

In the rest of the paper, we shall deal with the case of interest for ODEs, that is, $E \subset \mathbb{R}^{m+1}$ and $f: E \rightarrow \mathbb{R}^{m}$. From now on, $E \subseteq \mathbb{R}^{m+1}$ will always denote an r.e. open set, and $\left\{a_{n}\right\},\left\{r_{n}\right\}$ and $B\left(a_{n}, r_{n}\right)$ are the corresponding sequences in Definition 2.3. Also for notational convenience, we will sometimes simply write $f$ for $f(t, x)$ and refer to $t \in \mathbb{R}$ as the first argument and $x \in \mathbb{R}^{m}$ as the second argument of $f$.

It can be shown [24 that if $f$ is computable on $E$, then there exists a computable modulus function $e: \mathbb{N} \times \mathbb{N} \rightarrow \mathbb{N}$ which is locally effective in the sense that $|f(x)-f(y)| \leq 2^{-k}$ whenever $x, y \in \bigcup_{j=0}^{n} \overline{B\left(a_{j}, r_{j}\right)}$ and $|x-y| \leq 2^{-e(k, n)}$. In particular, this implies that $f$ must be continuous.

Definition 2.6. Let $E=\bigcup_{n=0}^{\infty} B\left(a_{n}, r_{n}\right) \subseteq \mathbb{R}^{l}$, where $\overline{B\left(a_{n}, r_{n}\right)} \subseteq E$, be an r.e. open set. A function $f: E \rightarrow \mathbb{R}^{m}$ is called effectively locally Lipschitz in the second argument if there exists a computable sequence $\left\{K_{n}\right\}$ of positive integers such that

$$
|f(t, x)-f(t, y)| \leq K_{n}|y-x| \text { whenever }(t, x),(t, y) \in \overline{B\left(a_{n}, r_{n}\right)} .
$$

Obviously an effectively locally Lipschitz function $f: E \rightarrow \mathbb{R}^{m}$ is also effectively locally Lipschitz in the second argument. On the other hand, denoting by $C^{k}(E)$ the space of $k$-fold continuously differentiable functions defined on $E$, it is trivially shown that if $f \in C^{1}(E)$, then $f$ is locally Lipschitz on $E$; in other words, continuous differentiability is a strictly stronger condition than that of being locally Lipschitz. This fact extends to computable functions in the following way.

Theorem 2.7. Assume that $f: E \rightarrow \mathbb{R}^{m}$ is a computable function in $C^{1}(E)$ (meaning that both $f$ and its derivative $f^{\prime}$ are computable). Then $f$ is effectively locally Lipschitz on E. 
Proof. Let $K_{n}$ be an integer greater than or equal to $\max _{x \in \overline{B\left(a_{n}, r_{n}\right)}}\left|f^{\prime}(x)\right|$. Since $f^{\prime}, a_{n}, r_{n}$ are computable, the real number $\max _{x \in \overline{B\left(a_{n}, r_{n}\right)}}\left|f^{\prime}(x)\right|$ is also computable (notice that, because $f^{\prime}$ is computable, it has a modulus of continuity that can be used to obtain the maximum over $\overline{B\left(a_{n}, r_{n}\right)}$ within any preassigned precision). Moreover, since $f^{\prime}$ has a locally effective modulus of continuity, we may assume that the sequence $\left\{K_{n}\right\}$ is a computable sequence of positive integers. Now, for any $x, y \in \overline{B\left(a_{n}, r_{n}\right)}$, let $u=y-x$. Then $x+s u \in \overline{B\left(a_{n}, r_{n}\right)}$ for $0 \leq s \leq 1$ because $\overline{B\left(a_{n}, r_{n}\right)}$ is a convex set. Define $F:[0,1] \rightarrow \mathbb{R}^{n}$ by $F(s)=f(x+s u)$. By the chain rule,

Therefore,

$$
F^{\prime}(s)=f^{\prime}(x+s u) \cdot u=f^{\prime}(x+s u) \cdot(y-x) .
$$

$|f(x)-f(y)|=|F(1)-F(0)|=\left|\int_{0}^{1} F^{\prime}(s) d s\right| \leq \int_{0}^{1}\left|f^{\prime}(x+s u) \cdot(y-x)\right| d s \leq K_{n}|x-y|$.

We now recall some basic results concerning initial-value problems defined with ODEs. Let us consider the following initial-value problem:

$$
\left\{\begin{array}{l}
\dot{x}=f(t, x) \\
x\left(t_{0}\right)=x_{0}
\end{array}\right.
$$

where $\left(t_{0}, x_{0}\right) \in E \subset \mathbb{R}^{m+1}$ and $f: E \rightarrow \mathbb{R}^{m}$ is a continuous function and satisfies a local Lipschitz condition in the second variable. The following is an immediate consequence of the fundamental existence-uniqueness theory for the initial-value problem (2.2) 8], 14] (the expressions $t \rightarrow \alpha^{+}$and $t \rightarrow \beta^{-}$mean that $t$ converges to $\alpha$ from above and to $\beta$ from below, respectively).

Theorem 2.8 (Maximal interval of existence). Let $E$ be an open subset of $\mathbb{R}^{m+1}$ and assume that $f: E \rightarrow \mathbb{R}^{m}$ is continuous on $E$ and locally Lipschitz in the second argument. Then for each $\left(t_{0}, x_{0}\right) \in E$, the problem (2.2) has a unique solution $x(t)$ defined on a maximal interval $(\alpha, \beta)$, on which it is $C^{1}$. The maximal interval is open and has the property that, if $\beta<+\infty$ (resp. $\alpha>-\infty)$, either $(t, x(t))$ approaches the boundary of $E$ or $x(t)$ is unbounded as $t \rightarrow \beta^{-}\left(\right.$resp. $\left.t \rightarrow \alpha^{+}\right)$.

\section{The MaXimal Interval IS R.E. OPEN}

Theorem 3.1. Let $E \subseteq \mathbb{R}^{m+1}$ be an r.e. open set and $f: E \rightarrow \mathbb{R}^{m}$ be a computable function that is also effectively locally Lipschitz in the second argument. Let $(\alpha, \beta)$ be the maximal interval of existence of the solution $x(t)$ of the initial-value problem (2.2), where $\left(t_{0}, x_{0}\right)$ is a computable point in $E$. Then $(\alpha, \beta)$ is an r.e. open interval and $x$ is a computable function on $(\alpha, \beta)$.

Proof. We consider the right maximal interval $\left(t_{0}, \beta\right)$ and prove $\left(t_{0}, \beta\right)$ is r.e. open and $x$ is computable on it. The same argument applies to the left maximal interval $\left(\alpha, t_{0}\right)$. For simplicity, we assume that $E$ is an open subset of $\mathbb{R}^{2}$.

Since $\left\{a_{n}\right\}$ and $\left\{r_{n}\right\}$ are computable sequences and $f$ is a computable function on $E$, both sequences $\left\{M_{n}\right\}, M_{n}=\max _{z \in \overline{B\left(a_{n}, r_{n}\right)}}|f(z)|+1$, and $\left\{K_{n}\right\}$ as defined in Definition 2.6 are computable, where $z=(t, x)$. A $\rho$-name of $z$ is used as an oracle in computations involving $z$ on oracle Turing machines.

Next we construct three (oracle) Turing machines, denoted as $T M_{1}, T M_{2}$ and $T M_{3}$. Let $T M_{1}$ be the Turing machine defined as follows: on any input $\left\{z_{k}\right\}_{k \in \mathbb{N}}$, 
where $\left\{z_{k}\right\}_{k \in \mathbb{N}}$ is a $\rho$-name of some $z \in E, T M_{1}$ computes $\left|z_{k}-a_{n}\right|$ and halts if $\left|z_{k}-a_{n}\right|<r_{n}-2^{-k+1}$. In this case, we say that the machine $T M_{1}$ halts at $(k, n)$. Obviously the machine will halt at any given $\rho$-name of every $z \in E$ and if the machine halts at $(k, n)$, then $B\left(z, 2^{-k}\right) \subset B\left(a_{n}, r_{n}\right)$. The output of $T M_{1}$, on input $\left\{z_{k}\right\}_{k \in \mathbb{N}}$, is the least (first) integer $\langle k, n\rangle$ such that $T M_{1}$ halts at $(k, n)$, where the following lexicographic ordering $\langle k, n\rangle$ is used for the pairs $(k, n)$ :

$\begin{array}{cccccccccc} & a_{1} & a_{2} & a_{3} & a_{4} & a_{5} & a_{6} & a_{7} & a_{8} & \ldots \\ z_{1} & 1 & 3 & 6 & 10 & 15 & 21 & 28 & \ldots & \\ z_{2} & 2 & 5 & 9 & 14 & 20 & 27 & \ldots & & \\ z_{3} & 4 & 8 & 13 & 19 & 26 & \ldots & & & \\ z_{4} & 7 & 12 & 18 & 25 & \ldots & & & & \\ z_{5} & 11 & 17 & 24 & \ldots & & & & & \\ z_{6} & 16 & 23 & \ldots & & & & & & \\ z_{7} & 22 & \ldots & & & & & & & \\ \vdots & & & & & & & & & \\ \vdots & & & & & & & & & \end{array}$

Let $T M_{2}$ be the Turing machine defined as follows: on any input $\rho$-name $\left\{z_{j}^{\prime}\right\}_{j \in \mathbb{N}}$ of $z^{\prime}=\left(t^{\prime}, x^{\prime}\right) \in E$, positive integers $M, K$ and $L$ satisfying $\overline{B\left(z^{\prime}, 2^{-L}\right)} \subset E$, $\max _{\left|z-z^{\prime}\right| \leq 2^{-L}}|f(z)|+1 \leq M$, and $\left|f\left(z_{1}\right)-f\left(z_{2}\right)\right| \leq K\left|x_{1}-x_{2}\right|$ for all $z_{1}=$ $\left(t, x_{1}\right), z_{2}=\left(t, x_{2}\right) \in \overline{B\left(z^{\prime}, 2^{-L}\right)}, T M_{2}$ outputs the solution of the initial-value problem

$$
\left\{\begin{array}{l}
\dot{x}=f(t, x), \\
x\left(t^{\prime}\right)=x^{\prime}
\end{array}\right.
$$

over the time interval $\left[t^{\prime}, t^{\prime}+c^{\prime}\right]$, where $c^{\prime}=2^{-L} / M . T M_{2}$ can be constructed by making use of the classical proof of Picard-Lindelöf's theorem. The following construction follows essentially the proof of Theorem 3.1 of [8]. Let $c^{\prime}=2^{-L} / M$ and denote by $I$ the interval $\left[t^{\prime}, t^{\prime}+c^{\prime}\right]$. Define the successive approximations $x_{k}$ on $I$ as follows: $x_{0}(t)=x^{\prime}$ and $x_{k+1}(t)=x^{\prime}+\int_{t^{\prime}}^{t} f\left(s, x_{k}(s)\right) d s$ for $k=0,1,2, \ldots$ By induction on $k$ it can be shown that every $x_{k}$ exists on $I, x_{k} \in C^{1}(I)$ (with the appropriate interpretation of derivatives at endpoints as one-sided limits), and $\left(t, x_{k}(t)\right) \in B\left(z^{\prime}, 2^{-L}\right)$ for all $t \in I$. Since $f$ is a computable function and both integration and primitive recursion are computable operators, the sequence $\left\{x_{k}\right\}$ of the successive approximations is computable from $\left(t^{\prime}, x^{\prime}\right)$ plus the positive integers $M, K$ and $L$. Also it can be shown that $x_{k}$ converges uniformly on $I$ to a continuous limit function $x$, which is the solution of the given initial-value problem. Moreover, an upper bound for the error in approximating the solution $x$ by the $k$ th approximation $x_{k}$ is easily calculable by the definition of $x_{k}$, and is given by $\left|x_{k}(t)-x(t)\right| \leq \frac{M}{K} \frac{\left(K c^{\prime}\right)^{k+1}}{(k+1) !} e^{K c^{\prime}}, t \in I$. It remains to show how $T M_{2}$ works. On any input $\rho$-name $\left\{z_{j}^{\prime}\right\}_{j \in \mathbb{N}}$ of $z^{\prime}=\left(t^{\prime}, x^{\prime}\right) \in E$, positive integers $M, K$ and $L$ satisfying the required conditions plus any $n \in \mathbb{N}$ (output accuracy) and any $\rho$-name of $t$ (input as an oracle), $t \in\left[t^{\prime}, t^{\prime}+c^{\prime}\right], T M_{2}$ first computes a $k \in \mathbb{N}$ such that $\frac{M}{K} \frac{\left(K c^{\prime}\right)^{k+1}}{(k+1) !} e^{K c^{\prime}} \leq 2^{-(n+1)}$. Then it computes a rational vector $r$ such that $\left|r-x_{k}(t)\right| \leq 2^{-(n+1)}$, and subsequently $|r-x(t)| \leq 2^{-n}$.

Let $T M_{3}$ be the Turing machine defined as follows: The input to $T M_{3}$ is the same as that to $T M_{2}$, the output of $T M_{3}$ is a $\rho$-name of $\left(t^{\prime}+c^{\prime}, x\left(t^{\prime}+c^{\prime}\right)\right) \in E$. 
Next we present an algorithm that computes a sequence $\left\{b_{l}\right\}_{l \in \mathbb{N}}$ converging to $\beta$ from below. Fix a $\rho$-name $\left\{z_{0 k}\right\}_{k \in \mathbb{N}}$ of $z_{0}=\left(t_{0}, x_{0}\right)$. On input $l$, set $j=0$. Now input $\left\{z_{0 k}\right\}$ into $T M_{1}$. Let $n=\left\langle k_{0}, n_{0}\right\rangle$ be the output of $T M_{1}$ on $\left\{z_{0 k}\right\}$. By the construction of $T M_{1}, B\left(z_{0}, 2^{-k_{0}}\right) \subset B\left(a_{n_{0}}, r_{n_{0}}\right)$. Next input $\left\{z_{0 k}\right\}_{k \in \mathbb{N}}, M_{n_{0}}, K_{n_{0}}, k_{0}$ into $T M_{2}$ and $T M_{3}$. Then $T M_{2}$ will output the solution of (2.2) over the time interval $\left[t_{0}, t_{0}+c_{0}\right]$, where $c_{0}=2^{-k_{0}} / M_{n_{0}}$. Denote this solution as $x^{0}:\left[t_{0}, t_{0}+\right.$ $\left.c_{0}\right] \rightarrow \mathbb{R}^{m}$. Separately, the machine $T M_{3}$ will output a $\rho$-name $\left\{z_{1 k}\right\}_{k \in \mathbb{N}}$ for $z_{1}=$ $\left(t_{0}+c_{0}, x^{0}\left(t_{0}+c_{0}\right)\right) \in E$. Now increase $j$ by 1 , i.e. set $j=1$. Repeat the above computation on the input $\left\{z_{1 k}\right\}_{k \in \mathbb{N}}$, i.e. input $\left\{z_{1 k}\right\}_{k \in \mathbb{N}}$ into $T M_{1}$. Let $n=\left\langle k_{1}, n_{1}\right\rangle$ be the output of $T M_{1}$. Next input $\left\{z_{1 k}\right\}, M_{n_{1}}, K_{n_{1}}$ and $k_{1}$ into $T M_{2}$ and $T M_{3}$. Then $T M_{2}$ will output the solution of the problem

$$
\left\{\begin{array}{l}
\dot{x}=f(t, x) \\
x\left(t_{0}+c_{0}\right)=x^{0}\left(t_{0}+c_{0}\right)
\end{array}\right.
$$

over the time interval $\left[t_{0}+c_{0}, t_{0}+c_{0}+c_{1}\right]$, where $c_{1}=2^{-k_{1}} / M_{n_{1}}$. Denote this solution as $x^{1}:\left[t_{0}+c_{0}, t_{0}+c_{0}+c_{1}\right] \rightarrow \mathbb{R}^{m}$. Also separately, the machine $T M_{3}$ will output a $\rho$-name $\left\{z_{2 k}\right\}_{k \in \mathbb{N}}$ of $z_{2}=\left(t_{0}+c_{0}+c_{1}, x^{1}\left(t_{0}+c_{0}+c_{1}\right)\right) \in E$. Now increase $j$ by 1 again, i.e. set $j=2$. Repeat the computation on the input $\left\{z_{2 k}\right\}_{k \in \mathbb{N}}$. Halt the computation on input $l$ the first time when $j>l$ and output $b_{l}=t_{0}+c_{0}+$ $c_{1}+\ldots+c_{l}$. Since $l \mapsto b_{l}$ is an input-output function of a Turing algorithm, the increasing sequence $\left\{b_{l}\right\}_{l \in \mathbb{N}}$ of rational numbers is a computable sequence. Also by the uniqueness of the solution of (2.2) and Pour-El/Richards' Patching Theorem [19, it follows that the map $x^{b_{l}}:\left[t_{0}, b_{l}\right] \rightarrow E, x^{b_{l}}(t)=x^{j}(t)$ if $t_{0}+c_{0}+\ldots+c_{j-1} \leq$ $t \leq t_{0}+c_{0}+\ldots+c_{j-1}+c_{j}, 0 \leq j \leq l$ with $c_{-1}=0$, is the solution of the initial-value problem (2.2) over the time interval $\left[t_{0}, b_{l}\right]$ and this solution is computable. Let $O=\bigcup_{l=0}^{\infty}\left(t_{0}, b_{l}\right]$ and $x: O \rightarrow E$ be the solution of the initial-value problem (2.2) over the time interval $O$. To complete the proof, we need to show that (a) $O$ is the right maximal interval of existence of the solution of (2.2); (b) $O$ is r.e. open; and (c) $x$ is computable on $O$. For simplicity, we take $t_{0}=0$.

To prove (a), assume that $O$ is not the maximal interval of existence of the solution of the problem (2.2). Then (2.2) has a solution on an interval $(0, \beta)$ with $O \varsubsetneqq(0, \beta)$. Choose $\beta^{\prime} \in(0, \beta) \backslash O$. Then $O \varsubsetneqq\left(0, \beta^{\prime}\right] \varsubsetneqq(0, \beta)$. Since $\left\{b_{l}\right\}_{l \in \mathbb{N}}$ is a monotonically increasing sequence bounded above by $\beta^{\prime}$, it converges to a limit $\gamma$ less than or equal to $\beta^{\prime}$. Since $\gamma \in(0, \beta),(\gamma, x(\gamma))$ must lie in $E$. Then there exists an $n$ such that $(\gamma, x(\gamma)) \in B\left(a_{n}, r_{n}\right)$. Moreover, there is also an integer $M$ such that $(\gamma, x(\gamma)) \in B\left((\gamma, x(\gamma)), 2^{-M+2}\right) \subset B\left(a_{n}, r_{n}\right)$. Since $x:\left[0, \beta^{\prime}\right] \rightarrow \mathbb{R}^{m}$ is continuous and $b_{l} \rightarrow \gamma$ as $l \rightarrow \infty$, it follows that $x\left(b_{l}\right) \rightarrow x(\gamma)$ as $l \rightarrow \infty$. Consequently there exists an integer $N$ such that

$$
\left(b_{N}, x\left(b_{N}\right)\right) \in B\left(\left(b_{N}, x\left(b_{N}\right)\right), 2^{-M+1}\right) \subset B\left((\gamma, x(\gamma)), 2^{-M+2}\right) \subset B\left(a_{n}, r_{n}\right)
$$

and

$$
b_{N}+\min _{0 \leq\langle i, j\rangle \leq\langle M+2, n\rangle} 2^{-i} / M_{j}>\gamma .
$$

We observe that for any $\rho$-name $\left\{y_{k}\right\}$ of $\left(b_{N}, x\left(b_{N}\right)\right)$,

$$
\begin{aligned}
\left|y_{M+2}-a_{n}\right| & \leq\left|y_{M+2}-\left(b_{N}, x\left(b_{N}\right)\right)\right|+\left|\left(b_{N}, x\left(b_{N}\right)\right)-a_{n}\right| \\
& <2^{-(M+2)}+r_{n}-2^{-M+1} \\
& =r_{n}-2^{-(M+2)+1}(4-1 / 2)<r_{n}-2^{-(M+2)+1} .
\end{aligned}
$$


By the construction of the machine $T M_{1}$ it follows that on any $\rho$-name of $\left(b_{N}, x\left(b_{N}\right)\right)$ as input, $T M_{1}$ will halt no later than $\langle M+2, n\rangle$. Thus $b_{N+1}=b_{N}+c_{N+1}$, where $c_{N+1}=2^{-i} / M_{j}$ for some $\langle i, j\rangle$ less than or equal to $\langle M+2, n\rangle$, which implies that $b_{N+1}>\gamma$. There is a contradiction because $\left\{b_{l}\right\}_{l \in \mathbb{N}}$ is an increasing sequence converging to $\gamma$. This completes the proof of (a). We now prove (b). Since by Theorem 2.8 the maximal interval of existence is open, $O$ is an open interval. It is also independent of the choice of $\rho$-names of $x_{0}$. By the proof of (a), it follows that $O=\bigcup_{l=0}^{\infty}\left(0, b_{l}\right]=\bigcup_{l=0}^{\infty}\left(0, b_{l}\right)$. Since $\left\{b_{l}\right\}_{l \in \mathbb{N}}$ is a computable sequence of rational numbers, $O$ is r.e. open by definition. Finally we prove (c), that is, $x$ is computable on $O$. Recall that $O=\bigcup_{l=0}^{\infty}\left(0, b_{l}\right)$ and $\left\{b_{l}\right\}_{l \in \mathbb{N}}$ is a computable sequence of rational numbers. For any $t \in O$, to compute $x(t)$, we first compute an $l \geq 0$ such that $t<b_{l}$ and then compute $x^{b_{l}}(t)$. By definition, $x(t)=x^{b_{l}}(t)$.

We mention that the above proof is effective in the sense that given $\left(E, f, t_{0}, x_{0}\right)$, one can compute $(\alpha, \beta, x), \beta$ from below and $\alpha$ from above; i.e. one can compute a sequence of rationals that converges to $\beta$ from below and a sequence of rationals that converges to $\alpha$ from above. However, the rate of the convergence might not be computable.

\section{Noncomputability of the maximal interval}

In this section, we present some noncomputability results concerning ODEs. In particular, we show that for the initial-value problem (2.2) defined by the computable data $f$ and $\left(t_{0}, x_{0}\right)$, the maximal interval may be noncomputable. We will present two versions of this result; the proof methods are different and the results are interesting on their own. In the first case, where only continuity is required, we can explicitly construct $f$ with a finite expression on bounded domains. For that reason, we prove a preliminary lemma. The second result is for the stronger case where $f$ is analytic, but lacks the finiteness feature: the function $f$ is defined as a (computable) power series.

4.1. Continuous case. Since a computable function is continuous 24, Theorem 2.8 ensures existence of the maximal interval of the following IVP (4.1).

Lemma 4.1. Let $a: \mathbb{N} \rightarrow \mathbb{N}$ be a computable function. Then there exists a computable and effectively locally Lipschitz function $f: \mathbb{R} \rightarrow \mathbb{R}$ such that the unique solution of the problem

$$
\left\{\begin{array}{l}
\dot{x}=f(x) \\
x(0)=0
\end{array}\right.
$$

is defined on a maximal interval $(-\alpha, \alpha)$ with

$$
\alpha=\sum_{i=0}^{\infty} \frac{1}{2^{a(i)}} .
$$

Proof. We only need to construct the function $f$. The idea is as follows: $f$ is constructed piecewisely on intervals of the form $[i, i+1], i \in \mathbb{N}$ (for negative values, we take $f(x)=f(|x|))$ in such a way that, for a fixed $i$, the solution of the initialvalue problem

$$
\dot{x}=f(x), x(0)=i
$$




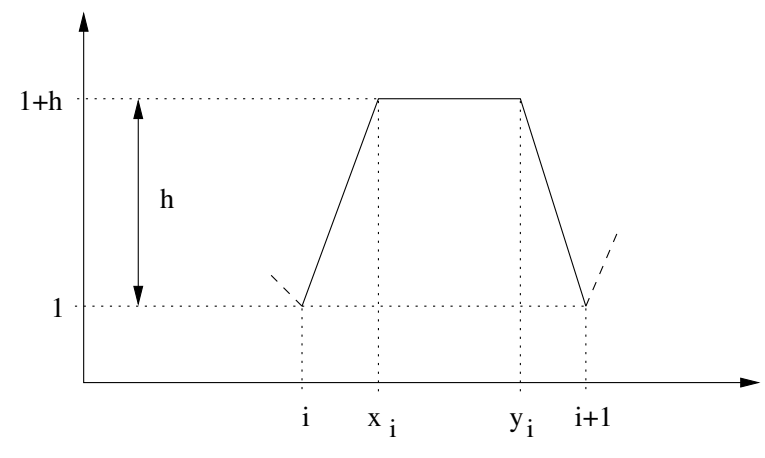

Figure 1. Sketch of the function $f$ on the interval $[i, i+1]$, for $i \in \mathbb{N}$.

satisfies $x\left(2^{-a(i)}\right)=i+1$, which implies that the solution of the problem $\dot{x}=f(x)$ and $x(0)=0$ will satisfy $x\left(2^{-a(0)}\right)=1, x\left(2^{-a(0)}+2^{-a(1)}\right)=2, \ldots$, or more generally,

$$
x\left(\sum_{i=0}^{n} 2^{-a(i)}\right)=n+1, \quad \text { for all } n \in \mathbb{N} .
$$

Notice that $f$ does not depend on $t$ and therefore the solution is invariant under time translations. If we take $\alpha=\sum_{i=0}^{\infty} 2^{-a(i)}$, then $x(t) \rightarrow \infty$ as $t \rightarrow \alpha^{-}$. For $t<0$, since we required $f(x)=f(|x|)$, (4.2) implies that $x\left(-2^{-a(i)}\right)=-(i+1)$, and hence $x(t) \rightarrow-\infty$ as $t \rightarrow-\alpha^{+}$. Therefore the maximal interval must be $(-\alpha, \alpha)$.

We now construct the desired function $f$ on intervals of the form $[i, i+1], i \in \mathbb{N}$. Since $f$ must be continuous, we need to glue the values of $f$ at the endpoints of these intervals. This is achieved by assuming that $f(i)=1$ for $i \in \mathbb{N}$ (in principle, the value of 1 is rather arbitrary; however, some singularities may arise when we consider other values, e.g. 0).

The function $f$ is defined on each interval $[i, i+1]$ as suggested by Fig. 1 , $f$ is piecewise linear and consists of three line segments, which meet at points $x_{i}=$ $x\left(t_{i, 1}\right)$ and $y_{i}=x\left(t_{i, 2}\right)$, with $0<t_{i, 1}<t_{i, 2}<2^{-a(i)}$ and $i=x(0)<x_{i}<y_{i}<i+1$. The points $x_{i}$ and $y_{i}$ are to be defined. For the moment let us assume that $a(i) \geq 1$. The more general case $a(i) \geq 0$ will be dealt with later in the proof. We now define the function $f$ on the interval $[i, i+1]$ as follows:

$$
f(x)= \begin{cases}1+(x-i) 2^{a(i)} /\left(x_{i}-i\right) & \text { if } x \in\left[i, x_{i}\right), \\ 1+2^{a(i)} & \text { if } x \in\left[x_{i}, y_{i}\right), \\ 1+2^{a(i)}-\left(x-y_{i}\right) 2^{a(i)} /\left(i+1-y_{i}\right) & \text { if } x \in\left[y_{i}, i+1\right) ;\end{cases}
$$

i.e. assume that $h=2^{a(i)}$ in Fig. 1. Supposing that $x_{i}$ and $y_{i}$ are equidistant from $i$ and $i+1$, respectively, and assuming that the solution $x(t)$ of the initial-value problem $\dot{x}=f(x), x(0)=i$ satisfies $x\left(2^{-a(i)}\right)=i+1$, then one obtains the following values for $x_{i}$ and $y_{i}$ :

where

$$
x_{i}=i+\frac{1-\Delta_{i}}{2}, \quad y_{i}=i+\frac{1+\Delta_{i}}{2},
$$

$$
0<\Delta_{i}=\frac{2^{-a(i)}-2^{-a(i)} \ln \left(2^{a(i)}+1\right)}{\left(1+2^{a(i)}\right)^{-1}-2^{-a(i)} \ln \left(2^{a(i)}+1\right)}<1 .
$$


It remains to treat the general case where $a(i) \geq 0$. This can be easily done as follows. First define the recursive function $a^{\prime}: \mathbb{N} \rightarrow \mathbb{N}$ by $a^{\prime}(i)=a(i)+1$. Using the previous result, we can construct an IVP $\dot{x}=f(x), x(0)=0$ with maximal interval $\left(-\alpha^{\prime}, \alpha^{\prime}\right)$, where

$$
\alpha^{\prime}=\frac{1}{2} \alpha, \quad \alpha=\sum_{i=0}^{\infty} \frac{1}{2^{a(i)}}
$$

Thus, if in the previous problem time is slowed down by a linear factor of $1 / 2$, i.e., the change of independent variables $\tilde{t}=t / 2$ is performed, we arrive at an IVP $\dot{x}=f(x) / 2, x(0)=0$ whose maximal interval of existence is $(-\alpha, \alpha)$.

Theorem 4.2 (Continuous case). There exists a continuous computable and effectively locally Lipschitz function $f: \mathbb{R} \rightarrow \mathbb{R}$ such that the unique solution of the problem

$$
\left\{\begin{array}{l}
\dot{x}=f(x) \\
x(0)=0
\end{array}\right.
$$

is defined on a noncomputable maximal interval.

Proof. In [19, Sec. 0.2], it is shown that if $a: \mathbb{N} \rightarrow \mathbb{N}$ is a one-to-one recursive function generating a recursively enumerable nonrecursive set $A$, then $\alpha=\sum_{i=0}^{\infty} 2^{-a(i)}$ is a noncomputable real number. Consequently, the open interval $(-\alpha, \alpha)$ is noncomputable. The theorem now follows immediately from the previous lemma.

The function $f$ in Theorem 4.2 can be constructed so that $f$ is of class $C^{\infty}$ and all its derivatives are computable functions (just "smooth" the "corners"). This condition matches the assumption set down in Theorem 3.1. Thus, Theorem 3.1 gives rise to the best possible result concerning computability of a maximal interval for smooth functions.

4.2. Analytic case. We now show that the result of Section 4.1 can be strengthened to cover the case of computable analytic functions.

Lemma 4.3. Let $a: \mathbb{N} \rightarrow \mathbb{N}$ be a one-to-one recursive function generating a recursively enumerable nonrecursive set. Then there is a computable analytic function $\varphi$ with the following properties:

(1) $\varphi$ is defined on $(-\alpha, \alpha)$, where $\alpha=\sum_{i=0}^{\infty} 2^{-a(i)}$ is a noncomputable real [19];

(2) $\varphi(x) \rightarrow \pm \infty$ as $x \rightarrow \pm \alpha^{\mp}$;

(3) $\varphi:(-\alpha, \alpha) \rightarrow \mathbb{R}$ is odd and bijective.

Proof. Define $\varphi$ as

$$
\varphi(x)=\sum_{n=0}^{\infty} a_{n} x^{n}, \quad a_{n}= \begin{cases}\left(\sum_{i=0}^{n} 2^{-a(i)}\right)^{-n} & \text { if } n \text { is odd } \\ 0 & \text { if } n \text { is even }\end{cases}
$$


The radius of convergence of this function is given by

$$
R=\frac{1}{\varlimsup_{n \rightarrow \infty} \sqrt[n]{a_{n}}}=\sum_{i=0}^{\infty} 2^{-a(i)}=\alpha .
$$

Moreover, one has $a_{2 n+1}>1 / \alpha^{2 n+1}$, which in turn implies

$$
\varphi(x)=\sum_{n=0}^{\infty} a_{2 n+1} x^{2 n+1}>\sum_{n=0}^{\infty}\left(\frac{x}{\alpha}\right)^{2 n+1} .
$$

Therefore, $\varphi(x) \rightarrow+\infty$ as $x \rightarrow \alpha^{-}$. Since $\varphi$ is odd by construction, it follows that $\varphi(x) \rightarrow-\infty$ as $x \rightarrow-\alpha^{+}$. Note also that

$$
\varphi^{\prime}(x)=\sum_{n=0}^{\infty}(n+1) a_{n+1} x^{n}
$$

and thus $\varphi^{\prime}(x)>0$ for all $x \in(-\alpha, \alpha)$ (all coefficients $(n+1) a_{n+1}$ are nonnegative, and only even powers have nonzero coefficients). This implies that $\varphi$ is injective and therefore bijective according to condition (2) of the statement. It also follows from (4.3) and our choice of $a_{n}$ that $\varphi^{\prime}$ is strictly increasing on $[0, \alpha)$ and, since $\varphi^{\prime}$ is even, decreasing on $(-\alpha, 0]$.

It remains to show that $\varphi$ is computable. Assume, without loss of generality, that $x \geq 0$. Since $a: \mathbb{N} \rightarrow \mathbb{N}$ is computable by assumption, there is a TM that, for any input $k \in \mathbb{N}$ (output precision) and any $x \in(-\alpha, \alpha)$ with $x \geq 0$, computes first a rational number $\varepsilon>0$ satisfying $0 \leq x<\alpha-\varepsilon$, then an $n(k) \in \mathbb{N}$ satisfying $\sum_{i=0}^{n(k)} 2^{-a(i)}>x+\varepsilon$ and $\left(\frac{x}{x+\varepsilon(x)}\right)^{n(k)} \frac{(x+\varepsilon(x))^{2}}{(x+\varepsilon(x))^{2}-x^{2}}<2^{-k-1}$. We observe that

$$
\begin{aligned}
& \left|\varphi(x)-\sum_{i=0}^{n(k)} a_{i} x^{i}\right|=\sum_{i=n(k)+1}^{\infty} a_{i} x^{i}=\sum_{i=\left\lceil\frac{n(k)}{2}\right\rceil}^{\infty}\left(\frac{x}{\sum_{j=0}^{2 i+1} 2^{-a(j)}}\right)^{2 i+1} \\
\leq & \sum_{i=\left\lceil\frac{n(k)}{2}\right\rceil}^{\infty}\left(\frac{x}{x+\varepsilon(x)}\right)^{2 i+1} \leq\left(\frac{x}{x+\varepsilon(x)}\right)^{n(k)} \frac{(x+\varepsilon(x))^{2}}{(x+\varepsilon(x))^{2}-x^{2}} \leq 2^{-k-1} .
\end{aligned}
$$

Then, if our TM computes a rational $r_{k}$ satisfying

$$
\left|r_{k}-\sum_{i=0}^{n(k)} a_{i} x^{i}\right| \leq 2^{-k-1}
$$

one concludes that $\left|r_{k}-\varphi(x)\right| \leq 2^{-k}$. Thus $\varphi$ is computable.

Theorem 4.4. There exists an analytic computable function $f: \mathbb{R} \rightarrow \mathbb{R}$ such that the unique solution of the problem

$$
\dot{x}=f(x), x(0)=0
$$

is defined on a noncomputable maximal interval.

Proof. Define the function $\varphi$ as in the previous lemma. By the lemma, $\varphi^{\prime}(x)>0$ for all $x \in(-\alpha, \alpha)$, and consequently $\varphi^{-1}$ exists over $\mathbb{R}$. Denote $\psi=\varphi^{-1}$. Then

$$
\psi^{\prime}(x)=\frac{1}{\varphi^{\prime}(\psi(x))}>0 \quad \text { for all } x \in \mathbb{R} .
$$


Similarly, $\varphi^{\prime}(x)=\left(\psi^{\prime}(\varphi(x))\right)^{-1}$ for all $x \in(-\alpha, \alpha)$, and therefore $\varphi$ is the solution of the IVP

$$
\left\{\begin{array}{l}
\dot{y}=f(y) \\
y(0)=0
\end{array}\right.
$$

where $f: \mathbb{R} \rightarrow \mathbb{R}$ is defined by $f(x)=1 / \psi^{\prime}(x)$ (note that $f$ is defined for all real numbers due to (4.4)). Since $\psi$ (and thus $\psi^{\prime}$ ) is analytic and computable (see e.g. 24]), so is $f$.

\section{Boundedness is Undecidable}

In the previous section it is shown that, in general, given an IVP (1.1) and $t>t_{0}$, we cannot devise an algorithm that tells us how close we are from one of the endpoints of the maximal interval. Nevertheless, this does not rule out the existence of an algorithm that can determine some partial information about the maximal interval. A further question of interest is if there exists an algorithm that can decide whether a given analytic IVP has a bounded maximal interval. As we now show, the answer to this question is also negative.

Theorem 5.1. Given an IVP (2.2) with maximal interval $(\alpha, \beta)$, where $f$ is analytic, $f$ and $\left(t_{0}, x_{0}\right)$ are computable, there is no effective (i.e. computable) procedure to determine whether $\beta<\infty$ or $\beta=\infty$.

Proof. Suppose that there is an effective procedure that determines whether $\beta<\infty$ or $\beta=\infty$, i.e. there is a Turing machine $T M_{1}$ that with input $\left\langle f, t_{0}, x_{0}\right\rangle$ returns 1 if $\beta<\infty$ and 0 otherwise $\left(f, t_{0}, x_{0}\right.$ mean the canonical encodings of the machines computing $f, t_{0}$, and $x_{0}$, respectively; see 24 for further details), where $\left\langle f, t_{0}, x_{0}\right\rangle$ is the data defining the IVP $\dot{x}=f(t, x)$ and $x\left(t_{0}\right)=x_{0}$. Consider the following undecidable problem [16], Th. II.2.3: "Let $\psi: \mathbb{N}^{2} \rightarrow \mathbb{N}$ be the function generated by a Universal Turing machine. Then, given $i \in \mathbb{N}$, decide if $\psi(i, i)$ is defined". Let $T M_{2}$ be a Turing machine that computes $\psi$. Define the recursive function $g: \mathbb{N}^{2} \rightarrow \mathbb{N}$ by

$$
g(i, j)= \begin{cases}0 & \text { if } T M_{2} \text { halts with input }(i, i) \text { in } \leq j \text { steps } \\ 1 & \text { otherwise }\end{cases}
$$

Note that

$$
\psi(i, i) \text { is defined iff } \exists j_{0} \in \mathbb{N} \quad\left(\forall j \geq j_{0}, g(i, j)=0\right) .
$$

Next consider the sequence of functions $\left\{\varphi_{i}\right\}$, where $\varphi_{i}: \mathbb{R} \rightarrow \mathbb{R}$ is defined by

$$
\varphi_{i}(x)=\sum_{n=1}^{\infty} a_{i, 2 n} x^{2 n}, \quad \text { where } a_{i, n}=\left(\frac{1}{3}\right)^{n^{2}}+\left(\frac{1}{3}\right)^{n} g(i, n) .
$$

Following arguments similar to those of section 4.2, one concludes that: (i) $\varphi_{i}$ is analytic and computable, (ii) the sequence $\left\{\varphi_{i}\right\}$ is computable by an oracle machine $T M_{3}$ since $g: \mathbb{N}^{2} \rightarrow \mathbb{N}$ is recursive, (iii) the radius of convergence of $\varphi_{i}$ is $+\infty$ iff $\psi(i, i)$ is defined, (iv) we can design a Turing machine $T M_{4}$ in the following way: on input $i \in \mathbb{N}, T M_{4}$ computes $\varphi_{i}^{\prime} \circ U_{1}^{2}$ and then runs $T M_{1}$ on the input $\left\langle\varphi_{i}^{\prime} \circ U_{1}^{2}, 0,0\right\rangle$ (note that, given some $i \in \mathbb{N}$, the "code" for $\varphi_{i}^{\prime}$ can be obtained from the "code" for $\varphi_{i}$ that, in turn, can be obtained from the "code" for $T M_{3}$ ), where $\varphi_{i}^{\prime}$ is the derivative of $\varphi_{i}$ and $U_{1}^{2}: \mathbb{R}^{2} \rightarrow \mathbb{R}$ is the projection function defined by $U_{1}^{2}(t, x)=t$. 
Recall that $\left\langle\varphi_{i}^{\prime} \circ U_{1}^{2}, 0,0\right\rangle$ is the data defining the IVP $\dot{x}=\varphi_{i}^{\prime}(t)$ and $x(0)=0$. Then by (5.1), (iii) and the design of $T M_{4}$, we arrive at the following conclusion:

$$
T M_{4} \text { on input } i \text { outputs } \begin{cases}0 & \text { if } \psi(i, i) \text { is defined, } \\ 1 & \text { otherwise, }\end{cases}
$$

i.e. $T M_{4}$ decides an undecidable problem, and we have a contradiction.

\section{ACKNOWLEDGMENT}

The authors thank the anonymous referee for the helpful comments and suggestions.

\section{REFERENCES}

1. O. Aberth, Computable analysis and differential equations, Intuitionism and Proof Theory (A. Kino, J. Myhill, and R.E. Vesley, eds.), Studies in Logic and the Foundations of Mathematics, North-Holland, Amsterdam, 1970, pp. 47-52. MR0276087 (43:1835)

2. - The failure in computable analysis of a classical existence theorem for differential equations, Proc. Amer. Math. Soc. 30 (1971), 151-156. MR0302982 (46:2124)

3. E. Bishop and D. S. Bridges, Constructive analysis, Springer, 1985. MR804042 (87d:03172)

4. L. Blum, F. Cucker, M. Shub, and S. Smale, Complexity and real computation, Springer, 1998. MR.1479636 (99a:68070)

5. L. Blum, M. Shub, and S. Smale, On a theory of computation and complexity over the real numbers: NP-completeness, recursive functions and universal machines, Bull. Amer. Math. Soc. 21 (1989), no. 1, 1-46. MR974426 (90a:68022)

6. M. Braverman and S. Cook, Computing over the reals: Foundations for scientific computing, Notices Amer. Math. Soc. 53 (2006), no. 3, 318-329. MR2208383 (2006m:68019)

7. M. Braverman and M. Yampolsky, Non-computable Julia sets, Journal Amer. Math. Soc. 19 (2006), 551-578. MR 2220099 (2007m:37110)

8. E. A. Coddington and N. Levinson, Theory of ordinary differential equations, McGraw-Hill, 1955. MR0069338(16:1022b)

9. J. Denef and L. Lipshitz, Decision problems for differential equations, J. Symbolic Logic 54 (1989), no. 3, 941-950. MR1011182 (91b:03074)

10. A. Grzegorczyk, Computable functionals, Fund. Math. 42 (1955), 168-202. MR0086756 $(19: 238 b)$

11. J. Hauck, Ein Kriterium für die konstruktive Lösbarkeit der Differentialgleichung $y^{\prime}=f(x, y)$, Z. Math. Logik Grundlag. Math. 31 (1985), no. 4, 357-362. MR807518 (87b:03136)

12. K.-I Ko, Complexity theory of real functions, Birkhäuser, Boston, MA, 1991. MR.1137517 (93i:03057)

13. D. Lacombe, Extension de la notion de fonction récursive aux fonctions d'une ou plusieurs variables réelles III, Comptes Rendus de l'Académie des Sciences Paris 241 (1955), 151-153. MR0072080 (17:225e)

14. S. Lefschetz, Differential equations: Geometric theory, 2nd ed., Interscience, 1963. MR 0153903 (27:3864)

15. S. Mazur, Computable analysis, PWN, 1963. MR0153553 (27:3517)

16. P. Odifreddi, Classical recursion theory, vol. 1, Elsevier, 1989. MR.982269 (90d:03072)

17. M. B. Pour-El and J. I. Richards, A computable ordinary differential equation which possesses no computable solution, Ann. Math. Logic 17 (1979), 61-90. MR552416 (81k:03064)

18. _ The wave equation with computable initial data such that its unique solution is not computable, Adv. Math. 39 (1981), 215-239. MR614161 (83e:03101)

19. Computability in analysis and physics, Springer, 1989. MR1005942 (90k:03062)

20. M. B. Pour-El and N. Zhong, The wave equation with computable initial data whose unique solution is nowhere computable, Math. Log. Quart. 43 (1997), 499-509. MR1477618 (98m:03097)

21. K. Ruohonen, An effective Cauchy-Peano existence theorem for unique solutions, Internat. J. Found. Comput. Sci. 7 (1996), no. 2, 151-160.

22. M. Sipser, Introduction to the theory of computation, PWS Publishing Company, Boston, MA, 1997. 
23. A. M. Turing, On computable numbers, with an application to the Entscheidungsproblem, Proc. London Math. Soc. 2 (1936), no. 42, 230-265.

24. K. Weihrauch, Computable analysis: An introduction, Springer-Verlag, 2000. MR 1795407 (2002b:03129)

25. K. Weihrauch and N. Zhong, Is wave propagation computable or can wave computers beat the Turing machine?, Proc. London Math. Soc. 85 (2002), no. 3, 312-332. MR.1912053 (2003i:03064)

DM/FCt, Universidade do Algarve, C. Gambelas, 8005-139 Faro, Portugal - and SQIG, Instituto de Telecomunicações, Instituto Superior Técnico, Torre Norte, Piso 10, Av. Rovisco Pais, 1049-001 Lisboa, Portugal

E-mail address: dgraca@ualg.pt

Department of Mathematical Sciences, University of Cincinnati, Cincinnati, Ohio 45221-0025

E-mail address: ning.zhong@uc.edu

Camgsd and Dep. Matemática, Faculdade de Ciências, Ed C6, Piso 2, Campo Grande, 1749-006 Lisboa, Portugal

E-mail address: jbuescu@ptmat.fc.ul.pt 